\title{
Vas deferens invasion: A neglected issue in the sampling of radical prostatectomy materials
}

\author{
Nuri Yigit, MD; Yildirim Karslioglu, MD; Bulent Kurt, MD \\ Gulhane Military Medical Academy and School of Medicine, Department of Pathology, Ankara, Turkey
}

Cite as: Can Urol Assoc J 2014;8(5-6):e554-7. http://dx.doi.org/10.5489/cuaj.1802 Published online August 11, 2014.

\section{Abstract}

A radical prostatectomy affects the prostate, bilateral seminal vesicles (SV), and the distal parts of the bilateral vasa deferentia (VD). SV invasion (SVI) is associated with an increased risk of lymph node metastasis and recurrence. However, the significance of VD invasion (VDI), either with or without the involvement of their surgical margins, has not been fully appreciated. We think VDI might have an independent prognostic significance, as does SVI, and should be incorporated into the pathology guidelines and the staging systems of prostatic adenocarcinoma. Our case illustrates this.

\section{Introduction}

It is well-known that seminal vesicle invasion (SVI) is associated with an increased risk of lymph node metastasis and recurrence. ${ }^{1,2} \mathrm{SVI}$ is reported between $3.3 \%$ and $22.6 \%$ in some latest studies, ${ }^{3-6}$ and it is designated as pT3b in the American Joint Committee on Cancer (AJCC) TNM staging system. ${ }^{7}$ While a thorough surgical margin sampling of the VD has long been practiced, the potential meaning and the possible impacts on the prognosis of mural tumour invasion to the VD has not yet been studied and appraised independently.

Extraprostatic muscular layer involvement of SV is considered the most important and exclusive hallmark of SVI,

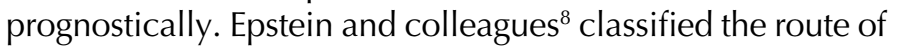
the tumour to the SV. Tumour extension from the base of the prostate into the periseminal vesicle tissue, with subsequent invasion into the muscular wall of the SV and by way of the ejaculatory ducts into the SV are two common types of invasion. 9,10 To us, it is logical that these invasion patterns are also possible for VD invasion because of its proximity to the SV and the anatomical connection with ejaculatory ducts, as well as the similar histological structure with SV. Despite the recommendations of the surgical margin sampling, we did not come across any obvious description or even any comments dealing with the mural VD invasion in English language literature. It was a bit surprising to us, and we think that this is mostly because of the lack of awareness among clinicians and pathologists.

Furthermore, there is much debate on the necessity and the importance of VD margin reporting. The latest pathology reporting guideline for prostatic carcinoma, issued by College of American Pathologists (CAP), does not include any information and recommendation about VD sampling. ${ }^{11}$ On the contrary, the Association of Directors of Anatomic and Surgical Pathology (ADASP) has offered sampling and reporting of the VD surgical margin, routinely. Yet, they also did not comment on the meaning of a positive VD surgical margin, and its potential effects on the patient's follow-up. ${ }^{12}$

On the other hand, the sampling of the VD margins was considered an optional procedure according to the declaration of the International Society of Urological Pathologists (ISUP) consensus conference in 2011, notwithstanding the considerable $(46 \%)$ opposition claiming that it should be done routinely. ${ }^{13}$

\section{Case report}

A 69-year-old man was admitted to the urology department with a long history of urinary complaints, including hematuria, dysuria and urinary frequency. Digital rectal examination confirmed an enlarged prostate. His total prostate-specific antigen (PSA) level was $2.13 \mathrm{ng} / \mathrm{mL}$. A transrectal ultrasoundguided needle core biopsy was performed. Upon microscopic examination, acinar adenocarcinoma with Gleason score $7(3+4)$ was observed in most of the biopsy cores. Subsequently, the patient was underwent a radical prostatectomy.

Macroscopically, the prostate seemed enlarged, measuring $5 \times 4.2 \times 4 \mathrm{~cm}$. The cut surfaces were grayish-yellow 
coloured, firm and somewhat nodular throughout the gland. The prostate, bilateral SV and VD were totally submitted and processed. Histopathological evaluation revealed an extensive tumour, which infiltrated all the posterior right and left quadrants of the prostate. The Gleason score was consistent with the prior biopsy (Fig. 1, part A). As a not-so-frequent finding, the tumour infiltrated to the ejaculatory ducts and the muscular parts of the bilateral extraprostatic SVs (Fig. 1, part B). On close inspection, we noted that the tumour was also invading the muscular layer up to the adventitia of the left VD in its middle part, without involvement of the clamped surgical margin (Fig. 1, part C to F). Extraprostatic extension (EPE), extraseminal or extravasal extension was not detected, and the circumferential surgical margins were all clear. Postoperatively, the total PSA levels were $0.03 \mathrm{ng} / \mathrm{mL}$ and $0.1 \mathrm{ng} /$ $\mathrm{mL}$ at month 3 and 15 , respectively. To date, the patient is well with no evidence of recurrence.

\section{Discussion}

Radical prostatectomy specimens need to be carefully examined macroscopically and histologically so that prognostically important parameters, such as surgical margin positivity, EPE, SVI, and the status of the bladder neck, are not overlooked. Among these parameters, surgical margins, EPE, and SVI are well-known and described in most guidelines and staging systems. However, the status of vasa deferentia has been omitted, despite the fact that they are important extraprostatic neighboring structures which are very similar to SV.

The neoplastic lesions of VD are extremely rare. Most of the primary VD tumours are of mesenchymal origin and benign in nature. Primary malignant VD tumours are so rare that they can be regarded as absent. Based on the literature, secondary tumoural infiltration of distal VD, mostly caused by prostate and bladder tumours, are more commonly encountered than those of any other types of tumours. ${ }^{14}$
The status of the SV is an essential part of the radical prostatectomy report, because the SVI is associated with an increased risk of lymph node metastasis and recurrence..$^{1,2}$ Therefore, there is literature on the frequency of SVI, reported between $3.3 \%$ and $18 \% .^{3-6}$ In the 7 th Edition of AJCC TNM staging system, SVI has been recognized as an independent prognostic factor and designated as pT3b. ${ }^{7}$

We think that the distal parts of VD, which are the integral elements of the radical prostatectomy specimens, deserve a lot more attention. Unfortunately, it can hardly be said that there is a standardized way to sample and process of the VD. Some guidelines and protocols deal with the VD as a generic method of surgical margin sampling. To our knowl-
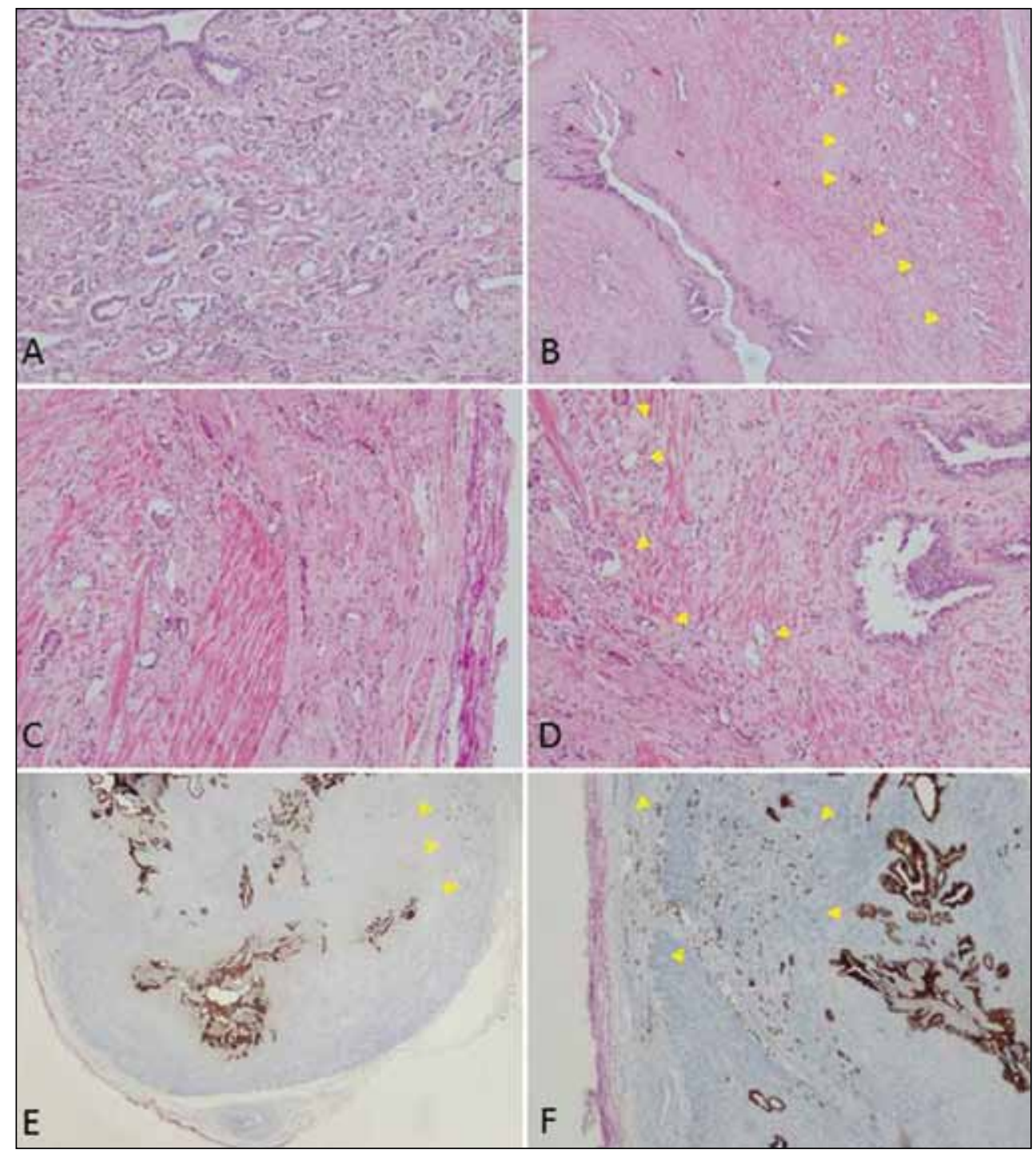

Fig. 1. (A) Acinar adenocarcinoma in prostate with Gleason grade 3 pattern (Hematoxylin and eosin stain [H\&E] $\times 100)$. (B) Involvement of the muscular layer of the extraprostatic seminal vesicles (arrowheads) (H\&E, $\times 40)$. (C, D) Tumour invades up to the adventitia and the muscular part of the left vasa deferentia (VD) in non-surgical margin areas (arrowheads) (H\&E, both $\times 100)$. (E, F) Immunohistochemically, pancytokeratin antibody demonstrates the invasion of the VD clearly (arrowheads) $(\times 20, \times 40)$. 
Yigit et al.

edge, there is no recommendation related to the handling of VD, except for their surgical margins. For this reason, there is no way to document the real frequency and prognostic significance of the tumours.

Billis and colleagues investigated a series of 105 cases undergoing radical prostatectomy and reported 7 (6.67\%) VDI at surgical margin. ${ }^{15}$ If the authors would have submitted the total VD, then we would have seen higher rates of $\mathrm{VDI}$, in addition to the involvement of surgical margins alone. Five of these 7 patients, bilateral involvements for both VD and SV were detected. Among the remaining, 1 had unilateral for VD and SV, and surprisingly the other patient had bilateral VDI with unilateral SVI. We think that this observation is noteworthy, because it can be concluded that VDI might be an independent phenomenon from the SVI, a kind of skip involvement sparing the SV. In the same study, 5 of these 7 patients showed recurrent and/or metastatic disease in a follow-up period of 7 to 39 months. They also concluded that these cases significantly correlated with SVI, extensive involvement of prostate by tumour, EPE, higher Gleason scores (7-10), and positive bladder neck margins.

Nguyen and colleagues investigated 875 patients with clinically organ-confined prostate cancer who underwent extended pelvic lymph node dissection and radical retropubic prostatectomy. They observed a $5 \%$ local recurrence rate, some with skeletal metastases ${ }^{16}$ In their study, about $22 \%$ of the locally recurrent cases recurred the site of the VD surgical margin. Considering the main anatomical locations of the tumours and the positive surgical margins in the prostatectomy materials, the sites of the local recurrences were not well-correlated to the primary areas of the tumour involvement. In some cases, although positive surgical margins were found at the prostatic apex, local recurrences were detected at the resection sites of the VD.

Our patient had prostatic acinar adenocarcinoma. The tumour was extensive and infiltrated both SV. At first, we found no remarkable features; however, when we examined the VD, which were totally submitted in addition to their surgical margins, we noted that the tumour also invaded the left VD. After multiple serial sections and immunohistochemical studies using pancytokeratin (Pan CK), PSA, and alpha-methylacyl-CoA racemase (AMACR) antibodies, we found that the surgical margin of the VD was tumour-free. In this patient, the VDI was detectable because of our tendency to submit not just the surgical margins, but all VD segments, routinely. To date, after 21 months of follow-up, the patient is well with no evidence of recurrence.

Our case raises the question of whether the VDI has any independent prognostic impact as the SVI. Unfortunately, the answer requires a universal way of sampling of VD in radical prostatectomy materials in larger series; this seems improbable to realize in the archival materials due to a lack of standard sampling practices. Therefore, the potential meaning and the possible impacts on the prognosis of mural tumour invasion to the VD, either accompanying a positive or negative surgical margin, cannot currently be appreciated. We can emphasize the need for guideline clarification in radical prostatectomy sampling. In this way, the total sampling and the processing of VD, in addition to their surgical margins, might become standard practice.

\section{Conclusion}

If the tumour is able to spread along the ejaculatory sinus, extraprostatic soft tissues and the adventitia of SV, as well as the discontinuous infiltration to the muscular wall of the $\mathrm{SV}$, then the tumour can also spread to the VD. However, the consistency of VD is quite different and harder than the neighboring structures and the periprostatic tissues. Therefore, it can be concluded that the muscular layer of $\mathrm{VD}$, as a spreading route for the tumour, has much more resistance for invasion, just like the arterial walls. Based on this assumption, a tumour invading the VD is highly unlikely to have an indolent biological behaviour and clinical course. As such, the presence of VDI in addition to the surgical margins deserves to be emphasized in pathology reports. It is known that a widespread VDI via ejaculatory ducts without surgical margins can be seen in the tumours with or without EPE and SVI. So, another reason for the inclusion of this finding in pathology reports is to construct a knowledge base which can be used to assess its possible prognostic effects. This requires a standardized way of the total sampling.

We think that keeping these points in mind while dealing with the radical prostatectomy materials might contribute to this area of surgical pathology and provide better care to patients with prostatic adenocarcinoma.

Competing interests: Dr. Yigit, Dr. Karslioglu and Dr. Kurt all declare no competing financial or personal interests.

This paper has been peer-reviewed.

\section{References}

1. Bader $P$, Burkhard $F C$, Markwalder R, et al. Disease progression and survival of patients with positive lymph nodes after radical prostatectomy. Is there a chance of cure? J Urol 2003;169:849-54. http:// dx.doi.org/10.1097/01.ju.0000049032.38743.c7

2. Masterson TA, Pettus JA, Middleton RG, et al. Isolated seminal vesicle invasion imparts better outcomes after radical retropubic prostatectomy for clinically localized prostate cancer: prognostic stratification of pT3b disease by nodal and margin status. Urology 2005;66:152-5. http://dx.doi.org/10.1016/i. urology.2005.01.014 
3. Pierorazio PM, Ross AE, Schaeffer EM, et al. A contemporary analysis of outcomes of adenocarcinoma of the prostate with seminal vesicle invasion (pT3b) after radical prostatectomy. J Urol 201 1;185:1691-7. http://dx.doi.org/10.1016/i.juro.2010.12.059

4. Bastide C, Rossi D, Lechevallier E, et al. Seminal vesicle invasion: What is the best adjuvant treatment after radical prostatectomy? BJU Int 2012;109:525-30; discussion 531-2. http://dx.doi.org/10.1111/ j.1464-410X.2011.10332.x

5. Sapre N, Pedersen J, Hong MK, et al. Re-evaluating the biological significance of seminal vesicle invasion (SVI) in locally advanced prostate cancer. BJU Int 2012;110(Suppl 4):58-63. http://dx.doi. org/10.1111/j.1464-410X.2012.11477.x

6. Kristiansen $A$, Wiklund $F$, Wiklund $P$, et al. Prognostic significance of patterns of seminal vesicle invasion in prostate cancer. Histopathology 2013;62:1049-56. http://dx.doi.org/10.1111/his.12104

7. Edge SB, Byrd DR, Carducci MA, et al. AJCC Cancer Staging Manual. 7th edition. New York, NY: Springer; 2009.

8. Epstein JI, Partin AW, Potter SR, et al. Adenocarcinoma of the prostate invading the seminal vesicle: Prognostic stratification based on pathologic parameters. Urology 2000;56:283-8.

9. Samaratunga H, Samaratunga D, Perry-Keene J, et al. Distal seminal vesicle invasion by prostate adenocarcinoma does not occur in isolation of proximal seminal vesicle invasion or lymphovascular infiltration. Pathology 2010;42;330-3. http://dx.doi.org/10.3109/00313021003767330

10. Billis A, Teixeira DA, Stelini RF, et al. Seminal vesicle invasion in radical prostatectomies: Which is the most common route of invasion? Int Urol Nephrol 2007;39:1097-102. http://dx.doi.org/10.1007/ s1 1255-007-9189-7
11. Srigley JR, Humphrey PA, Amin MB, et al. Protocol for the examination of specimens from patients with carcinoma of the prostate gland. Arch Pathol Lab Med 2009;133:1568-76.

12. Epstein JI, Srigley J, Grignon D, et al.; Association of Directors of Anatomic and Surgical Pathology. Recommendations for the reporting of prostate carcinoma: Association of Directors of Anatomic and Surgical Pathology. Am J Clin Pathol 2008;129:24-30. http://dx.doi.org/10.1309/59U8R6N5R7BKCWLV

13. Berney DM, Wheeler TM, Grignon DJ, et al; ISUP Prostate Cancer Group. International Society of Urological Pathology (ISUP) Consensus Conference on Handling and Staging of Radical Prostatectomy Specimens. Working group 4: seminal vesicles and lymph nodes. Mod Pathol 2011;24:39-47. http://dx.doi. org/10.1038/modpathol.2010.160

14. Kim B, Kawashima A, Ryu JA, et al. Imaging of the seminal vesicle and vas deferens. Radiographics 2009;29:1105-21. http://dx.doi.org/10.1148/rg.294085235

15. Billis A, Freitas LLL, Magna LA. Vas deferens involvement in radical prostatectomies. Prevalence and significance. United States and Canadian Academy of Pathology (USCAP) Annual Meeting. Chicago, IL, Feb 2002. Abstract 647, 155A.

16. Nguyen DP, Giannarini $G$, Seiler $R$, et al. Local recurrence after retropubic radical prostatectomy for prostate cancer does not exclusively occur at the anastomotic site. BJU Int 2013;112:E243-9. http://dx.doi. org/10.1111/j.1464-410X.2012.11506.x

Correspondence: Dr. Nuri Yigit, Department of Pathology, Gulhane Military Medical Academy and School of Medicine, 06010 Etlik, Ankara, Turkey; nyigit@gata.edu.tr 